\title{
Pulmonary infiltrates in non-HIV immunocompromised patients: a diagnostic approach using non-invasive and bronchoscopic procedures
}

\author{
A Rañó, C Agustí, P Jimenez, J Angrill, N Benito, C Danés, J González, M Rovira,
} T Pumarola, A Moreno, A Torres

\begin{abstract}
Background-The development of pulmonary infiltrates is a frequent life threatening complication in immunocompromised patients, requiring early diagnosis and specific treatment. In the present study non-invasive and bronchoscopic diagnostic techniques were applied in patients with different non-HIV immunocompromised conditions to determine the aetiology of the pulmonary infiltrates and to evaluate the impact of these methods on therapeutic decisions and outcome in this population.
\end{abstract}

Servei de
Pneumologia, Institut Clínic de Pneumología i Cirurgía Toràcica, Hospital Clínic de Barcelona, Villarroel 170, 08036 Barcelona, Spain

A Rañó

C Agustí

P Jimenez

J Angrill

A Torres

Servei de

Microbiologia i

Malalties Infeccioses,

Institut Clínic de

Infeccions i Immunitat

$\mathrm{N}$ Benito

C Danés

J González

T Pumarola

A Moreno

Servei d'Hematologia, Institut d'Hematologia i Oncologia

M Rovira

Departament de Medicina, Institut d'Investigacions Biomèdiques August Pi i Sunyer (IDIBAPS), Hospital Clinic de Barcelona, Barcelona, Spain

Correspondence to: Dr A Torres

atorres@medicina.ub.es

Received 9 August 2000 Returned to authors 21 September 2000 Revised version received

19 January 2001

Accepted for publication

22 January 2001
Methods-The non-invasive diagnostic methods included serological tests, blood antigen detection, and blood, nasopharyngeal wash (NPW), sputum and tracheobronchial aspirate (TBAS) cultures. Bronchoscopic techniques included fibrobronchial aspirate (FBAS), protected specimen brush (PSB), and bronchoalveolar lavage (BAL). Two hundred consecutive episodes of pulmonary infiltrates were prospectively evaluated during a 30 month period in 52 solid organ transplant recipients, 53 haematopoietic stem cell transplant (HSCT) recipients, 68 patients with haematological malignancies, and 27 patients requiring chronic treatment with corticosteroids and/or immunosuppressive drugs.

Results-An aetiological diagnosis was obtained in $162(81 \%)$ of the 200 patients. The aetiology of the pulmonary infiltrates was infectious in $125(77 \%)$ and noninfectious in $37(23 \%)$; $38(19 \%)$ remained undiagnosed. The main infectious aetiologies were bacterial $(48 / 125,24 \%)$, fungal $(33 / 125,17 \%)$, and viral $(20 / 125,10 \%)$, and the most frequent pathogens were $\mathrm{As}$ pergillus fumigatus $(\mathrm{n}=29)$, Staphylococcus aureus ( $\mathrm{n}=17)$, and Pseudomonas aeruginosa $(n=12)$. Among the noninfectious aetiologies, pulmonary oedema $(16 / 37,43 \%)$ and diffuse alveolar haemorrhage $(10 / 37,27 \%)$ were the most common causes. Non-invasive techniques led to the diagnosis of pulmonary infiltrates in $41 \%$ of the cases in which they were used; specifically, the diagnostic yield of blood cultures was 30/191 (16\%); sputum cultures 27/88 (31\%); NPW 9/50 (18\%); and TBAS $35 / 55(65 \%)$. Bronchoscopic techniques led to the diagnosis of pulmonary infiltrates in $59 \%$ of the cases in which they were used: FBAS 16/28 (57\%), BAL 68/135 (51\%), and PSB 30/125 (24\%). The results obtained with the different techniques led to a change in antibiotic treatment in $\mathbf{9 3}$ cases $(46 \%)$. Although changes in treatment did not have an impact on the overall mortality, patients with pulmonary infiltrates of an infectious aetiology in whom the change was made during the first 7 days had a better outcome $(29 \%$ mortality) than those in whom treatment was changed later $(71 \%$ mortality; $\mathbf{p}=\mathbf{0 . 0 0 1}$ ).

Conclusions-Non-invasive and bronchoscopic procedures are useful techniques for the diagnosis of pulmonary infiltrates in immunocompromised patients. Bronchial aspirates (FBAS and TBAS) and BAL have the highest diagnostic yield and impact on therapeutic decisions.

(Thorax 2001;56:379-387)

Keywords: immunocompromised; lung infection; bronchoalveolar lavage; nasopharyngeal wash; tracheobronchial aspirate; diagnosis

The good results obtained with solid organ transplants and haematopoietic stem cell transplantation (HSCT) in recent years have led to an increase in the number of potential candidates. ${ }^{1}$ One of the most important limiting factors for these treatments is the development of life threatening pulmonary complications. $^{2}$ Opportunistic and bacterial infections are common causes of pulmonary complications in immunocompromised patients including transplant recipients, ${ }^{34}$ and must be distinguished from other conditions such as pulmonary oedema, diffuse alveolar haemorrhage, malignant diseases, and pulmonary drug reactions. ${ }^{5-8}$ Accurate and prompt diagnosis of potentially treatable pulmonary complications is important in patients with severe immunosuppression.

In 1989 our group published the results of a protocol for the diagnosis of pulmonary infiltrates in a population of immunocompromised patients. ${ }^{9}$ In this study it was suggested that bronchoalveolar lavage (BAL) and protected specimen brush (PSB) techniques used concurrently were useful for the diagnosis of pulmonary complications in these patients. Over the last decade significant developments 
in diagnostic techniques have emerged. ${ }^{10}$ Furthermore, the administration of new and effective treatments has changed the pattern of pulmonary complications in immunocompromised patients. ${ }^{11-15}$ Recent publications have questioned the usefulness of bronchoscopic techniques in the diagnosis of pulmonary infiltrates in immunocompromised patients, suggesting that the information provided does not change treatment or improve survival and can cause significant complications. ${ }^{1617}$ In this study we have reappraised prospectively the current diagnostic usefulness and clinical impact of fibrobronchial aspiration (FBAS), BAL, and PSB for the study of pulmonary infiltrates in patients with different immunocompromised conditions (excluding HIV infected patients). We have also included other non-invasive procedures such as blood antigen detection, sputum, tracheobronchial aspirates (TBAS), and nasopharyngeal wash (NPW) to examine their diagnostic usefulness in this group of patients.

\section{Methods}

PATIENTS

We prospectively evaluated episodes of pulmonary infiltrates in 200 consecutive patients. All of the immunosuppressed patients with pulmonary infiltrates seen at a tertiary hospital between February 1998 and June 2000 were included (table 1). Patients included in the study belonged to four different groupsgroup 1: solid organ transplant (21 renal, 11 cardiac, 14 liver, and six pancreaticorenal) recipients; group 2: haematopoietic stem cell transplant (HSCT) recipients $(n=53)$; group 3: patients with haematological malignancies treated with chemotherapy $(n=68)$; and group 4: patients requiring chronic treatment with corticosteroids (minimum $30 \mathrm{mg}$ prednisone daily for the previous 30 days before inclusion) or immunosuppressive agents (azathioprine or cyclophosphamide; $n=27$ ). The underlying haematological malignancies of groups 2 and 3 were acute myeloid leukaemia $(n=30,25 \%)$, acute lymphoblastic leukaemia $(n=15,13 \%)$, Hodgkin's lymphoma $(\mathrm{n}=14,12 \%)$, chronic lymphoid leukaemia $(n=12,10 \%)$, chronic myeloid leukaemia $(\mathrm{n}=11,10 \%)$, and others $(n=39,30 \%)$. Fifty nine of the 200 infiltrates $(30 \%)$ were evaluated while the patients were on mechanical ventilation.
DATA COLLECTION

The following variables were recorded: age, sex, underlying immunosuppressed condition, leucocyte and platelet counts, serum biochemistry, arterial oxygen tension $\left(\mathrm{PaO}_{2}\right)$ /inspiratory oxygen fraction $\left(\mathrm{FiO}_{2}\right)$ ratio at inclusion, and APACHE II score (Acute Physiology and Chronic Health Evaluation) at the time of admission to hospital. The requirement for mechanical ventilation was also registered and survival was defined as hospital discharge.

DIAGNOSTIC PROCEDURES

Within the first 24-48 hours after the identification of the pulmonary infiltrates, samples of blood were drawn for blood cultures and serological and antigen testing. A sample of spontaneous or induced sputum (after nebulisation with 3\% saline solution) was obtained. A Gram stain was performed to assess the quality of the sample. ${ }^{18}$ A sample of NPW was taken for viral detection and tissue culture. In patients under mechanical ventilation, a sample of TBAS was obtained via an orotracheal tube under sterile conditions using a 22 inch 14 Fr suction catheter and collected into a mucus reservoir (Mocstrap; Proclinics, Barcelona, Spain). Antigen detection of Streptococcus pneumoniae (urine), Aspergillus sp (serum), and Chlamydia pneumoniae (pharyngeal swab), and DNA detection of Mycobacterium tuberculosis by ligase chain reaction (respiratory samples) were included once the study had been started.

Fibreoptic bronchoscopy (FOB) was performed before antibiotic treatment was started in 14 cases ( $9 \%$ of total FOB) and $48-96$ hours after starting antibiotic treatment in the remaining 136. Patients were not eligible for bronchoscopic exploration if arterial $\mathrm{PaO}_{2}$ was less than $7.3 \mathrm{kPa}$ with oxygen administration or if the platelet count was less than $50 \times 10^{6} / \mu 1$. The bronchoscope was passed transnasally or transorally into the trachea after topical nasal anaesthesia was instilled. In mechanically ventilated patients the bronchoscope was passed into the trachea through the endotracheal tube. A PSB sample (Microbiology Brush; Mill-Rose Laboratory Inc, Mentor, Ohio, USA $)^{19}$ was first obtained in 125 cases (62\%). Respiratory secretions were aspirated through the suction channel of the bronchoscope in 28 patients. Bronchoalveolar lavage was carried out with $150 \mathrm{ml}$ of sterile saline solution in three $50 \mathrm{ml}$

Table 1 Underlying immunosuppressed condition and clinical characteristics of the 200 patients studied

\begin{tabular}{llllll}
\hline & HSCT & $\begin{array}{l}\text { Haematological } \\
\text { malignancies }\end{array}$ & $\begin{array}{l}\text { Solid organ } \\
\text { transplantation }\end{array}$ & CIS & Total \\
\hline No. (\%) & $53(27 \%)$ & $68(34 \%)$ & $52(26 \%)$ & $27(13 \%)$ & 200 \\
Mean (SD) age (years) & $40(12)$ & $54(17)$ & $52(16)$ & $60(14)$ & $50(17)$ \\
M/F & $33 / 20$ & $36 / 32$ & $43 / 9$ & $13 / 14$ & $125 / 75$ \\
Neutropenia, n (\%) & $21(40 \%)$ & $28(41 \%)$ & $3(6 \%)$ & $1(2 \%)$ & $53(27 \%)$ \\
Mean (SD) APACHE II score & $18(7)$ & $19(8)$ & $20(8)$ & $20(8)$ & $19(8)$ \\
Mean (SD) PaO Paio $_{2}$ at inclusion & $269(102)$ & $266(95)$ & $221(126)$ & $222(113)$ & $249(109)$ \\
Mechanical ventilation requirement, n (\%) & $24(45 \%)$ & $23(34 \%)$ & $33(63 \%)$ & $15(56 \%)$ & $95(47 \%)$ \\
Mortality, n (\%) & $26(49 \%)$ & $21(31 \%)$ & $20(38 \%)$ & $14(52 \%)$ & $81(40 \%)$
\end{tabular}

HSCT $=$ haematopoietic stem cell transplantation; CIS = chronic immunosuppressive treatment; APACHE II = Acute Physiology and Chronic Health Evaluation II score at admission.

Underlying diseases: chronic obstructive pulmonary disease $(n=6)$, scleroderma $(n=3)$, idiopathic pulmonary fibrosis ( $n=4)$, systemic vasculitis $(n=3)$, rheumatoid arthritis $(n=2)$, glomerulonephritis $(n=2)$, and one each of systemic lupus erythematosus, relapsing polychondritis, dermatomyositis, mixed cryoglobulinaemia, Good-Pasteur syndrome, Beçhet syndrome, and primary biliary cirrhosis.

Neutropenia defined as a neutrophil count $<1 \times 10^{9}$ cells $/ 1$. 
aliquots in 135 patients (70\%). The lavage was performed in the involved lobe in patients with localised pulmonary infiltrates and in the middle lobe or lingula in patients with diffuse pulmonary infiltrates.

Although the primary intention was to perform all the different diagnostic procedures, the ultimate decision as to which of the procedures should be performed was always determined by the clinical state of the patient and the criteria of the physician in charge.

OTHER DIAGNOSTIC TECHNIQUES

Transbronchial (TBB) and bronchial biopsy specimens were obtained in 11 and three cases, respectively, and analysed pathologically and microbiologically. Videothoracoscopic (VTC) lung biopsy was carried out in two cases in which no diagnosis was reached by bronchoscopic examination. Necroscopic examination was performed in 19 of the 81 patients who died.

SAMPLE PROCESSING

Staining methods

Viral antigens in NPW and BAL samples were detected by a direct immunofluorescent staining method using a monoclonal antibody pool (influenza viruses $\mathrm{A}$ and $\mathrm{B}$, parainfluenza viruses 1, 2 and 3, respiratory syncytial virus, adenovirus, virus herpes simplex, and enterovirus). Sputum, FBAS and TBAS specimens were directly stained using Gram and ZiehlNeelsen methods. PSB and BAL smears were Gram and Giemsa stained for intracellular or extracellular bacterial and fungal detection and cellular differential count. In BAL samples additional stains were used: Ziehl-Neelsen staining for mycobacteria; Gomori staining for Pneumocystis carinii; PAS, haematoxylin-eosin, and Papanicolau for cell atypia; Pearls' staining for hemosiderophages.

\section{Cultures}

Sputum samples were qualitatively cultured for bacterial pathogens, fungi, and mycobacteria. Bronchoscopic samples were homogenised and processed for quantitative culture by serial dilutions (1:1 and $1: 10$, inoculating $0.1 \mathrm{ml} /$ plate) for bacterial pathogens. Culture media for Legionella spp and fungi were plated undiluted. FBAS, TBAS, and BAL specimens were also cultured for mycobacteria, and NPW and BAL samples were cultured for viruses on monolayers of Hep-2, A-549, MRC-5, and MDCK cell lines (Vircell, Granada, Spain). Cultures were maintained for 4 weeks at $37^{\circ} \mathrm{C}$ and examined regularly for a cytopathic effect. Viral growth was confirmed by immunofluorescence staining with monoclonal antibodies (Respiratory Pannel 1, Viral Screening \& Identification Kit, Light Diagnostics, Temecula, USA; Clonab CMV, Biotest, Germany).

Negative bacterial cultures were discarded after 3 days, Legionella after 10 days, mycobacteria after 6 weeks, and fungal cultures after 4 weeks. Positive bacterial cultures were counted as colony forming units per $\mathrm{ml}(\mathrm{cfu} / \mathrm{ml})$, and identification and susceptibility tests were performed according to standard methods.
Rapid diagnostic tests

The urinary antigen test for detection of $L$ pneumophila serogroup 1 was performed using an enzyme immunoassay (EIA) method (Biotest Legionella Urine Antigen EIA, Biotest, Germany) and $S$ pneumoniae was detected using an immunochromatography method (Binax Now $S$ pneumoniae Urinary Antigen Test, Binax, USA). Detection of the pp65 cytomegalovirus (CMV) antigen in BAL fluid and serum was performed using a specific monoclonal antibody (Clonab CMV, Biotest, Germany) by means of an immunocytochemistry method (APAAP; Dako, Denmark). Detection of Aspergillus spp galactomannan antigen in the serum was performed using an EIA method (Platelia Aspergillus, Sanofi Diagnostics Pasteur, France) and Mycobacterium tuberculosis DNA in respiratory samples was studied using a ligase chain reaction method (Abbot LCX Mycobacterium tuberculosis assay, Abbot Laboratories, Abbot Park, USA). C pneumoniae DNA was detected in nasopharyngeal swabs by a PCR-EIA method (Diasorin; Italy).

\section{DIAGNOSTIC DEFINITIONS}

The aetiology of pulmonary infiltrates was established based on the results of the different diagnostic procedures performed, the response to the specific treatment, and the histopathological evaluation of biopsy samples when available.

The aetiology of pulmonary infiltrates was defined as follows: (1) infectious: when there was a clinical suspicion of lower respiratory tract infection and a microbial agent was isolated in respiratory and/or non-respiratory samples; (2) non-infectious: when clinical data did not suggest an infectious aetiology, no microbiological agents were isolated in any processed sample, and the clinical course and response to treatment were in accordance with an alternative non-infectious aetiology; (3) undetermined (undiagnosed): when the pulmonary infiltrates did not meet criteria for infectious or non-infectious aetiologies.

\section{Diagnostic criteria for specific microorganisms}

Bacterial pneumonia was diagnosed whenever blood or sputum samples grew pathogenic bacteria. Similarly, when a quantitative bacterial culture from FBAS or TBAS, BAL or PSB specimens grew more than $10^{5}, 10^{4}$, or $10^{3} \mathrm{cfu} /$ $\mathrm{ml}$, respectively, the diagnosis of bacterial pneumonia was also established. Any growth of facultative pathogenic bacteria such as Streptococcus viridans and coagulase negative Staphylococcus was considered irrelevant.

Cytomegalovirus and other viruses were considered to be pathogens if they were isolated by cell culture from NPW or BAL specimens or when inclusion bodies were present on histopathological evaluation.

$P$ carinii pneumonia (PCP) was diagnosed by a positive Gomori methenamine silver stain. The identification of Legionella spp and mycobacteria was accepted as a definite diagnosis regardless of colony counts. Fungal pneumonia was diagnosed in the presence of a compatible clinical and radiographic pattern if fungal 
hyphae were identified by culture or by cytological evaluation of a Gram stain in at least two different respiratory samples, or when there was histological evidence for fungal pneumonia or positive blood cultures. ${ }^{20}$

Diagnostic criteria for non-infectious conditions Diffuse alveolar haemorrhage was diagnosed by established criteria ${ }^{5-7}$ and pulmonary oedema was diagnosed using clinical criteria. Bronchiolitis obliterans organising pneumonia (BOOP) was diagnosed histologically from samples obtained by transbronchial or open lung biopsies. Two patients were diagnosed with hypersensitivity pneumonitis secondary to bleomycin by clinical criteria and compatible radiological changes. In these patients the pulmonary infiltrates disappeared after bleomycin was withdrawn and corticosteroid treatment was started.

\section{ANTIMICROBIAL PROPHYLAXIS}

One hundred and thirteen patients $(57 \%)$ were receiving some type of antimicrobial prophylaxis at the onset of the pulmonary infiltrates: $43 \%$ in group $1,85 \%$ in group $2,55 \%$ in group 3 , and $33 \%$ in group 4 . The most frequent prophylaxis strategy was antibacterial treatment $(18 \%)$ followed by the combination of antiviral + antifungal + prophylaxis against PCP $(10 \%)$, followed by a combination of antibacterial + antifungal agents $(8 \%)$.

EMPIRICAL ANTIBIOTIC TREATMENT

The final decision regarding the initial antimicrobial treatment was taken by the clinician in charge. The most common combination consisted of a third generation cephalosporin plus an aminoglycoside. ${ }^{21}$ Alternative antibiotics were ureidopenicillins and/or ciprofloxacin. If Staphylococcus aureus infection was suspected or proven, vancomycin was added. Amphotericin B was added if no clinical or radiographic improvement was observed after 4-5 days of antibiotic treatment. Ganciclovir plus high dose immunoglobulin $G$ (or foscarnet as an alternative) were added if CMV infection was suspected or proven. G-CSF was occasionally given to patients with evidence of infection and prolonged neutropenia.

The initial antibiotic treatment was considered inadequate if the isolated microorganisms were not susceptible to or were not covered by the treatment administered. Changes in treatment were noted if they were based on either a positive or negative result from the noninvasive and bronchoscopic samples.

ANALYSIS OF DATA

Results are expressed as mean (SD). The median value (50th percentile) was used to evaluate the delay in establishing a specific diagnosis of the pulmonary infiltrates. All statistics were calculated using the SPSS statistical package (SPSS for Windows, version 9.0; Chicago, IL, USA). Categorical variables were compared using the $\chi^{2}$ test or Fisher's exact test when appropriate, and continuous variables were analysed using the non-parametric MannWhitney U test. A p value of $<0.05$ was considered statistically significant.

\section{Results}

AETIOLOGY OF PULMONARY INFILTRATES

A specific diagnosis was obtained in 162 of the 200 cases evaluated $(81 \%)$, with an infectious aetiology in 125 of the cases $(77 \%)$ and a noninfectious aetiology in $37(23 \%$, table 2). Bacteria were the most frequent microorganisms causing pulmonary infiltrates $(n=48,24 \%)$, followed by fungi $(n=33,17 \%)$ and viruses $(n=20,10 \%)$. In 15 cases $(7 \%)$ the aetiology of the pulmonary infiltrates was polymicrobial (table 3).

There were no statistically significant associations between the specific underlying immunosuppressive state and the aetiology of the pulmonary infiltrates.

\section{NON-INVASIVE TECHNIQUES}

Blood cultures were obtained from 191 cases and gave 30 positive results (16\%). When only patients with an infectious aetiology were considered, the diagnostic yield of blood cultures was $23 \%$. In 16 cases ( $7 \%$ ) blood cultures were the only means of diagnosis (most frequently due to isolation of Gram negative bacilli and MRSA).

Detection of pp65CMV antigen in peripheral blood mononuclear cells was performed in $91(45 \%)$ patients (47 HSCT recipients, 23 with haematological malignancies, and 21 solid organ transplant recipients) and was positive in seven of the eight cases of confirmed CMV pneumonitis. In three other cases with no evidence of CMV pneumonitis pp65CMV

Table 2 Aetiological diagnosis in relation to the underlying immunosuppressed condition

\begin{tabular}{|c|c|c|c|c|c|}
\hline & HSCT & $\begin{array}{l}\text { Haematological } \\
\text { malignancies }\end{array}$ & $\begin{array}{l}\text { Solid organ } \\
\text { transplantation }\end{array}$ & $C I S$ & Total \\
\hline Bacterial & $6(11 \%)$ & $16(24 \%)$ & $18(35 \%)$ & $8(30 \%)$ & $48(24 \%)$ \\
\hline Viral & $11(21 \%)$ & $6(9 \%)$ & $1(2 \%)$ & $2(7 \%)$ & $20(10 \%)$ \\
\hline Fungal & $7(13 \%)$ & $13(19 \%)$ & $6(12 \%)$ & $7(26 \%)$ & $33(17 \%)$ \\
\hline Polymicrobial & $3(6 \%)$ & $1(2 \%)$ & $9(17 \%)$ & $2(7 \%)$ & $15(7 \%)$ \\
\hline Other infectious aetiologies $\dagger$ & $1(2 \%)$ & $3(4 \%)$ & $1(2 \%)$ & $4(15 \%)$ & $9(5 \%)$ \\
\hline Pulmonary oedema & $3(6 \%)$ & $3(4 \%)$ & $10(19 \%)$ & - & $16(8 \%)$ \\
\hline DAH & $5(9 \%)$ & $2(3 \%)$ & $2(4 \%)$ & - & $10(5 \%)$ \\
\hline BOOP & $1(2 \%)$ & $2(3 \%)$ & - & $1(4 \%)$ & $4(2 \%)$ \\
\hline Other non-infectious aetiologies $\ddagger$ & $3(6 \%)$ & $4(6 \%)$ & - & - & $7(3 \%)$ \\
\hline Undetermined & $13(24 \%)$ & $17(25 \%)$ & $5(10 \%)$ & $3(11 \%)$ & $38(19 \%)$ \\
\hline
\end{tabular}

HSCT = haematopoietic stem cell transplantation; CIS = chronic immunosuppressive treatment; DAH = diffuse alveolar haemorrhage; $\mathrm{BOOP}=$ bronchiolitis obliterans organising pneumonia.

+Other infectious aetiologies include tuberculosis $(n=5), P$ carinii pneumonia $(n=4)$.

$\ddagger$ Other non-infectious entities include pulmonary involvement of Hodgkin’s disease $(n=3)$, drug toxicity due to bleomycin $(n=2)$, alveolar proteinosis $(n=1)$, and sarcoidosis $(n=1)$. 
Table 3 Infectious aetiologies in 125 patients diagnosed during lifetime or at necropsy

\begin{tabular}{|c|c|c|}
\hline Pathogens & $\begin{array}{l}\text { No of } \\
\text { cases }\end{array}$ & $\begin{array}{l}\text { No of } \\
\text { pathogens }\end{array}$ \\
\hline \multicolumn{3}{|l|}{ Bacterial } \\
\hline \multicolumn{3}{|l|}{ Gram positive } \\
\hline$S$ aureus & 12 & 17 \\
\hline$S$ pneumoniae & 2 & 5 \\
\hline Efecalis & 2 & 2 \\
\hline Efaecium & 1 & 1 \\
\hline$G$ morbillorum & 1 & 1 \\
\hline$S$ constellatum & 1 & 1 \\
\hline$N$ asteroides & 2 & 2 \\
\hline \multicolumn{3}{|l|}{ Gram negative } \\
\hline E coli & 6 & 7 \\
\hline P aeruginosa & 6 & 12 \\
\hline$S$ marcescens & 2 & 2 \\
\hline A baumanii & 2 & 3 \\
\hline$C$ freundii & 1 & 1 \\
\hline M morganii & 1 & 1 \\
\hline \multicolumn{3}{|l|}{ Other bacteria } \\
\hline M pneumoniae & 1 & 1 \\
\hline C pneumoniae & 2 & 2 \\
\hline L pneumophila & 2 & 2 \\
\hline \multicolumn{3}{|l|}{ Fungal } \\
\hline A fumigatus & 20 & 29 \\
\hline A niger & 1 & 1 \\
\hline A flavus & 1 & 1 \\
\hline C albicans & 8 & 12 \\
\hline C kruseii & 1 & 1 \\
\hline C tropicalis & 1 & 1 \\
\hline$S$ prolificans & 1 & 2 \\
\hline P purpurogenum & 1 & 1 \\
\hline \multicolumn{3}{|l|}{ Viral } \\
\hline Cytomegalovirus & 6 & 8 \\
\hline Influenza $\mathrm{A}$ virus & 5 & 8 \\
\hline RSV & 3 & 3 \\
\hline Parainfluenzae virus type 3 & 2 & 2 \\
\hline Virus herpes simplex (VHS)-1 & 1 & 1 \\
\hline Varicella zoster virus ${ }^{\star}$ & 1 & - \\
\hline Enterovirus & 1 & 1 \\
\hline \multicolumn{3}{|l|}{ Others } \\
\hline P carinii & 4 & 4 \\
\hline$M$ tuberculosis & 5 & 5 \\
\hline \multicolumn{3}{|l|}{ Mixed infections } \\
\hline Aspergillus $\mathrm{sp}+\mathrm{MRSA}$ & 3 & \\
\hline Aspergillus $\mathrm{sp}+P$ aeruginosa & 2 & \\
\hline E coli $+K$ pneumonia $+S$ maltophila & 1 & \\
\hline Aspergillus $\mathrm{sp}+\mathrm{CMV}$ & 1 & \\
\hline$C$ freundii $+P$ aeruginosa & 1 & \\
\hline MRSA $+E$ fecalis & 1 & \\
\hline$A$ baumanii $+P$ aeruginosa & 1 & \\
\hline A flavus + E faecium $+\mathrm{CMV}$ & 1 & \\
\hline A fumigatus $+S$ pneumoniae & 1 & \\
\hline A niger $+E$ coli + MRSA & 1 & \\
\hline A fumigatus + VHS-1 & 1 & \\
\hline $\begin{array}{l}\text { C tropicalis }+ \text { A fumigatus }+ \\
P \text { aeruginosa }\end{array}$ & 1 & \\
\hline
\end{tabular}

$\star$ Diagnosed by clinical criteria.

antigen was also positive. Serum Aspergillus spp galactomannan antigen detection was performed in $54(27 \%)$ patients (46 HSCT recipients or with haematological malignancies) and was positive in six of seven cases with confirmed pulmonary aspergillosis and in two without evidence of pneumonia by this pathogen. Two cases of $C$ pneumoniae, one case of $M$ pneumoniae, and one case of $L$ pneumoniae infection were diagnosed by serological tests and urinary antigen detection, respectively. One of the cases of $C$ pneumoniae was also confirmed by polymerase chain reaction (PCR) detection in the pharyngeal swab.

Nasopharyngeal wash was performed in 50 (25\%) patients (19 HSCT recipients, 26 with haematological malignancies, and four solid organ transplant recipients) and led to a definite diagnosis in nine (18\%) cases (table 4$)$.
All cases diagnosed by NPW were also diagnosed by BAL, except one case of RSV.

Sputum analysis was performed in 96 patients but only 88 samples were accepted for microbial analysis, 27 of which (22 spontaneous, five induced) resulted in a positive culture $(31 \%)$. When only the group of infectious pulmonary infiltrates was considered, this percentage increased to $46 \%$. Sputum culture was the only technique that gave a definite diagnosis in 10 cases $(8 \%)$.

Tracheobronchial aspirates (TBAS) were obtained in 55 patients on mechanical ventilation with 35 positive results $(64 \%)$. All of these were in the group with an infectious aetiology (table 4). In 11 cases (9\%) TBAS was the only technique that provided a definite diagnosis.

The overall diagnostic yield of non-invasive techniques was $40 \%$ and this percentage increased to $46 \%$ when we evaluated only those cases in whom the pulmonary infiltrates had an infectious aetiology.

\section{BRONCHOSCOPIC TECHNIQUES}

Fibrobronchial aspirates (FBAS) were obtained from 28 non-intubated patients and gave 16 positive results $(57 \%)$, with the isolation of a single microorganism in 15 cases and two in another case (table 4).

Protected specimen brush (PSB) specimens were obtained from 125 cases and cultures yielded high colony counts $\left(>10^{3} \mathrm{cfu} / \mathrm{ml}\right)$ of different microorganisms in 30 cases $(24 \%)$ (four cases with two simultaneous microorganisms) (table 4). Although the overall diagnostic yield for PSB was $24 \%$, and increased up to $43 \%$ in those with an infectious aetiology, in only one case was PSB the only technique to provide a definite diagnosis.

Bronchoalveolar lavage (BAL) was performed in 135 cases, giving an overall diagnostic yield of $51 \%(68 / 135)$ which increased to $69 \%(56 / 81)$ in those with an infectious aetiology (table 4). Furthermore, in 12 cases in which no microorganisms were detected, the recovered BAL fluid enabled a diagnosis to be made of a non-infectious cause of the pulmonary infiltrates: diffuse alveolar haemorrhage in 10 patients, one case of eosinophilia due to pulmonary toxicity secondary to the administration of bleomycin, and one case of alveolar proteinosis. In 26 cases $(20 \%)$ BAL was the only technique that provided a definite diagnosis.

Transbronchial biopsy (TBB) specimens were obtained from 11 selected cases and resulted in a specific diagnosis in six cases $(55 \%)$ : two cases of BOOP, two of pulmonary involvement of Hodgkin's disease, and two cases of bacterial pneumonia. Bronchial biopsy (BB) specimens were obtained in three patients and showed one case of $A$ fumigatus, one of $C$ tropicalis, and one of methicillin resistant $S$ aureus (MRSA) pneumonia.

Videothoracoscopic lung biopsy specimens were taken in two cases, resulting in the diagnosis of one case of pulmonary tuberculosis and one case of BOOP. 
Table 4 Pathogens isolated with the different techniques employed in respiratory samples*

\begin{tabular}{|c|c|c|c|c|c|c|c|}
\hline Pathogens & $\begin{array}{l}N P W \\
(n=50)\end{array}$ & $\begin{array}{l}\text { Sputum } \\
(n=88)\end{array}$ & $\begin{array}{l}F B A S \\
(n=28)\end{array}$ & $\begin{array}{l}T B A S \\
(n=55)\end{array}$ & $\begin{array}{l}P S B \\
(n=125)\end{array}$ & $\begin{array}{l}B A L \\
(n=135)\end{array}$ & $\begin{array}{l}\text { Total pathogens } \\
\text { isolated }\end{array}$ \\
\hline \multicolumn{8}{|l|}{ Bacterial } \\
\hline \multicolumn{8}{|l|}{ Gram positive } \\
\hline$S$ aureus & & 7 & 6 & 7 & 8 & 11 & 17 \\
\hline$S$ pneumoniae & & 3 & 1 & 3 & 1 & & 3 \\
\hline Efecalis & & & 1 & 1 & 1 & & 2 \\
\hline$N$ asteroides & & 2 & & & & & 2 \\
\hline \multicolumn{8}{|l|}{ Gram negative } \\
\hline$P$ aeruginosa & & 6 & 2 & 6 & 2 & 4 & 11 \\
\hline$S$ marcescens & & & & 1 & 1 & 2 & 2 \\
\hline A baumanii & & & & 1 & 1 & 3 & 3 \\
\hline$C$ freundii & & & & 1 & & & 1 \\
\hline M morganii & & & & & & 1 & 1 \\
\hline E coli & & 2 & & 1 & & & 1 \\
\hline$S$ maltophilia & & & & & 1 & 1 & 1 \\
\hline H influenzae & & 1 & & 1 & & 1 & 1 \\
\hline K pneumoniae & & & & 1 & & & 1 \\
\hline L pneumophila & & & & & 1 & 1 & 2 \\
\hline \multicolumn{8}{|l|}{ Fungal } \\
\hline A fumigatus & & 7 & 3 & 12 & 10 & 12 & 30 \\
\hline A flavus & & & & 1 & & & 1 \\
\hline$A$ niger & & & & & & 1 & 1 \\
\hline$C$ albicans & & 2 & 2 & 5 & 6 & 6 & 8 \\
\hline C tropicalis & & & & & & 1 & 1 \\
\hline C glabrata & & & & & & 1 & 1 \\
\hline P purpurogenum & & & & & & 1 & 2 \\
\hline$S$ prolificans & & & & 1 & 1 & 1 & 2 \\
\hline \multicolumn{8}{|l|}{ Viral } \\
\hline Cytomegalovirus & & & & & & 5 & 8 \\
\hline Influenza $\mathrm{A}$ virus & 5 & & & & & 8 & 8 \\
\hline RSV & 3 & & & & & 2 & 3 \\
\hline Parainfluenzae virus type 3 & & & & & & 2 & 2 \\
\hline Enterovirus & & & & & & 1 & 1 \\
\hline VHS-1 & 1 & & & & & 1 & 1 \\
\hline \multicolumn{8}{|l|}{ Others } \\
\hline Pcarinii & & & & & & 4 & 4 \\
\hline$M$ tuberculosis & & 2 & 1 & & & 1 & 5 \\
\hline
\end{tabular}

$\mathrm{NPW}=$ nasopharyngeal wash FBAS $=$ fibrobronchial aspirate; TBAS $=$ tracheobronchial aspirate PSB $=$ protected specimen brush; $\mathrm{BAL}=$ bronchoalveolar lavage; RSV = respiratory syncytial virus; VHS-1 = virus herpes simplex-1.

* Some organisms were simultaneously isolated with more than one technique and some of these techniques (FBAS, TBAS, PSB, and BAL) allowed the identification of more than one organism.

Necroscopic examination was performed in 19 of the 81 patients. Aspergillosis was identified in five cases and CMV pneumonitis in one, none of which had been diagnosed before death. Three cases of pulmonary aspergillosis (one with associated CMV pneumonitis not diagnosed before death), two of Pseudomonas $\mathrm{sp}$, three of CMV pneumonitis with diffuse alveolar damage, and one of $C$ tropicalis were confirmed. In four cases the necropsy showed diffuse alveolar damage with negative cultures.

OPTIMISATION OF PROCEDURES PERFORMED

With the aim of optimising the application of the different procedures included in the study,

Table 5 Impact of diagnostic techniques on the treatment of pulmonary infiltrates *

\begin{tabular}{lcc}
\hline Diagnostic technique & $\begin{array}{l}\text { No positive/ } \\
\text { no performed (\%) }\end{array}$ & $\begin{array}{l}\text { Modification of } \\
\text { treatment, } n \text { (\%) }\end{array}$ \\
\hline Blood cultures & $30 / 191(16 \%)$ & $4(5 \%)$ \\
CMV antigen detection & $11 / 91(12 \%)$ & $5(5 \%)$ \\
Aspergillus antigen detection & $8 / 54(15 \%)$ & $1(1 \%)$ \\
NPW & $9 / 50(18 \%)$ & $1(1 \%)$ \\
Sputum & $27 / 88(31 \%)$ & $11(12 \%)$ \\
FBAS & $16 / 28(57 \%)$ & $4(4 \%)$ \\
TBAS & $35 / 55(64 \%)$ & $24(26 \%)$ \\
PSB & $30 / 125(24 \%)$ & $2(2 \%)$ \\
BAL & $68 / 135(51 \%)$ & $35(38 \%)$ \\
TBB & $6 / 11(55 \%)$ & $4(4 \%)$ \\
VTC biopsy & $2 / 2(100 \%)$ & $2(2 \%)$ \\
\hline
\end{tabular}

$\mathrm{NPW}=$ nasopharyngeal wash; FBAS = fibrobronchial aspirate; TBAS = tracheobronchial aspirate; $\mathrm{PSB}=$ protected specimen brush; $\mathrm{BAL}=$ bronchoalveolar lavage; $\mathrm{TBB}=$ transbronchial biopsy; VTC $=$ videothoracoscopic.

*In some patients treatment changes were due to the results obtained with several diagnostic procedures simultaneously. we selected those 138 patients in whom a specific diagnosis was obtained using the noninvasive and bronchoscopic techniques, excluding those cases in whom the diagnosis was obtained by clinical criteria such as pulmonary oedema and those diagnosed by TBB, VTC, or necroscopic examination. In 61 of these 138 patients $(44 \%)$ it was possible to achieve the diagnosis using only non-invasive procedures (including blood, serological tests, and antigen detection in blood, NPW, sputum, and TBAS cultures). The remaining 91 diagnoses (66\%) required the use of different bronchoscopic procedures. No differences in clinical and radiographic characteristics were observed between the group of patients diagnosed by non-invasive procedures and those requiring bronchoscopic procedures. In the subgroup of patients diagnosed by means of bronchoscopic procedures, BAL had the highest diagnostic yield, providing $94 \%(65 / 69)$ of the diagnoses (including $100 \%$ of the non-infectious pulmonary infiltrates). Although PSB gave a diagnosis in $46 \%(29 / 63)$ of the population, all but one of the cases could also be diagnosed by BAL. The diagnostic role of FBAS cannot be properly ascertained in this population since the procedure was only performed in 19 patients, providing a diagnosis in $14(74 \%)$; however, in none of these 14 cases did the results of PSB add diagnostic information to that already obtained by FBAS. 
COMPLICATIONS

There were only three mild complications as a result of the FOB exploration: one patient with self-limited epistaxis, one with a moderate bronchial haemorrhage following transbronchial biopsy, and hypoxaemia requiring high flow oxygen by mask for 12 hours in a third patient.

IMPACT OF DIFFERENT DIAGNOSTIC TECHNIQUES ON TREATMENT OF PULMONARY INFILTRATES Empirical treatment was modified in 93 of the 200 cases (46\%) because of the results obtained with the different techniques used. Overall, non-invasive procedures caused a change in treatment in 45 of the 93 cases $(48 \%)$ whereas bronchoscopic techniques resulted in changes in 48 cases (52\%). In 19 cases it was the result of both non-invasive and bronchoscopic techniques that resulted in a change in treatment (table 5). BAL and TBAS had the greatest impact with treatment modifications in $38 \%(35 / 93)$ and $26 \%$ (24/93), respectively. The most common reason for changing treatment was the isolation of a microorganism not covered by the empirical treatment. This occurred in 56 cases $(60 \%)$ and was mostly attributable to isolation of Aspergillus sp, viruses, $M$ tuberculosis, and MRSA. In 18 cases $(20 \%)$ the reason for changing the empirical treatment was the isolation of a resistant strain. Finally, in 19 cases $(20 \%)$ the empirical antibiotic therapy was discontinued after diagnosing a noninfectious cause of pulmonary infiltrates (10 cases of diffuse alveolar haemorrhage, four of BOOP, two of pulmonary lymphoma, two of pulmonary toxicity resulting from bleomycin, and one of alveolar proteinosis).

OUTCOME

Eighty one of the 200 patients $(40 \%)$ included in the study died as a result of the pulmonary complication. Mortality in patients requiring mechanical ventilation was higher (73/95, $77 \%)$ than in those not requiring it $(8 / 105,7 \%$; $\mathrm{p}<0.0001$ ), being particularly high in HSCT recipients in whom the mortality rate reached $96 \%(26 / 27)$. Sixty four of the 125 patients with pulmonary infiltrates with an infectious aetiology died (51\%) compared with only six of the 37 patients with a non-infectious aetiology $(16 \% ; \mathrm{p}<0.0001)$. The mortality rate in patients in whom a specific diagnosis of the pulmonary infiltrates was established was $43 \%$ (70/162) compared with $29 \%(11 / 38)$ in those in whom a diagnosis could not be established during their lifetime $(\mathrm{p}=\mathrm{NS})$.

There was no relationship between the mortality rate and the specific aetiology of the pulmonary infiltrates. The mortality rates in patients with fungal, polymicrobial, viral, and bacterial pneumonia were $67 \%, 66 \%, 51 \%$, and $42 \%$, respectively ( $\mathrm{p}=\mathrm{NS}$ ). There were no deaths among patients with pulmonary tuberculosis, BOOP, or diffuse alveolar haemorrhage.

A significant difference in mortality was observed between patients in whom the diagnosis was established during the first 7 days (median value for the delay in establishing the diagnosis of the overall population) and those in whom the diagnosis was established later on. In the first group the mortality rate was $34 \%(29 / 85)$ compared with $53 \%(41 / 77)$ in those in whom the delay in diagnosis was longer than 7 days $(p=0.017)$. The observed difference in mortality depending on the delay in obtaining the diagnosis was probably related to the implications of an early change in empirical treatment. Thus, when we evaluated only patients requiring a change in empirical treatment $(n=93)$ we observed that those in whom this change was instituted during the first 7 days had a lower mortality rate $(14 / 42$, $30 \%$ ) than patients in whom the change was established later $(32 / 51,70 \% ; \mathrm{p}=0.007)$. This was particularly apparent in the 76 patients with pulmonary infiltrates with an infectious aetiology: mortality rate of $29 \%(13 / 34)$ in those in whom the pulmonary infection was diagnosed in the first 7 days compared with $71 \%(32 / 42)$ in patients in whom the pulmonary infection was diagnosed later $(p=0.001)$.

\section{Discussion}

This study shows that the simultaneous use of non-invasive and bronchoscopic procedures results in a specific diagnosis being made in most cases of pulmonary infiltrates in immunocompromised patients. Furthermore, obtaining a specific diagnosis early in the course of the disease allows the empirical treatment to be changed in a high percentage of cases (46\%) and may improve the survival of these patients. Using this diagnostic approach, we have diagnosed $81 \%$ of the episodes of pulmonary infiltrates, which compares favourably with $49 \%$ obtained 10 years ago by our group in a similar population. ${ }^{9}$

In our population the main cause of the pulmonary infiltrates was infection which constituted $77 \%$ of the episodes diagnosed. Bacterial infections and, specifically, those caused by $S$ aureus (mainly MRSA) and Gram negative bacilli (mainly P aeruginosa) were the most frequent infectious aetiologies found. Since bacteria represent the most prevalent threat to immunocompromised patients, each institution should define the empirical antibiotic treatment based on its own data. ${ }^{21-23}$

Fungal species, especially Aspergillus spp, represented the second most frequent infectious cause of pulmonary infiltrates in the present study. Death from Aspergillus pneumonia in immunosuppressed patients is exceedingly high, reaching $85 \%$ in some series. ${ }^{24}{ }^{25}$ In addition to the severe immunosuppression, this poor outcome may be partially explained by the delay in establishing the diagnosis and starting specific treatment. ${ }^{26}$ Although evidence of tissue invasion by fungi has classically been required to confirm the diagnosis, the presence of Aspergillus species in sputum or bronchial lavage cultures, ${ }^{27}$ as well as the detection of galactomannan fungal antigen, ${ }^{28}$ should be considered indicative of invasive disease until proved otherwise and warrants institution of specific therapy. In the present series Aspergillus pneumonia was diagnosed on 18 
occasions without tissue confirmation and antifungal treatment was instituted immediately, resulting in a mortality rate of $67 \%$. Whether this approach can improve the outcome of pulmonary aspergillosis remains to be demonstrated in well designed prospective series. The diagnosis of Candida pneumonia is even more controversial than Aspergillus pneumonia. ${ }^{20}$ Although we cannot exclude the possibility that some of the 12 cases of pulmonary candidiasis represent airway colonisation, three had tissue confirmation and five had positive blood cultures which further supported the diagnosis.

Viral pneumonia accounted for 20 episodes of pulmonary infiltrates, CMV being the most frequent aetiological agent. ${ }^{12}$ Evidence has been accumulating that community respiratory viruses such as respiratory syncytial virus, influenza virus, parainfluenza virus ${ }^{11} 13$ and adenovirus also play an important role in the aetiology of respiratory infiltrates in transplant recipients and that infection with these organisms may explain some of the pneumonic episodes previously classified as idiopathic. ${ }^{11}$ In the present series the rapid detection of parainfluenza virus in respiratory samples from two patients allowed us to initiate treatment with aerosolised ribavirin immediately, with a favourable outcome in both cases. So far, the role of any virus other than CMV or herpes simplex virus has been poorly defined. In the series published 10 years ago by our group, ${ }^{9}$ which included 113 episodes of pulmonary infiltrates in 93 immunosuppressed patients, viral investigations were restricted to the identification by culture of CMV and the cytopathological changes produced by CMV in cells recovered from the BAL fluid. Recently, new diagnostic tools such as quantitative PCR assays and fluorescein conjugated monoclonal antibodies have been developed, permitting the rapid detection of different viruses that are frequently associated with a high rate of morbidity and mortality. ${ }^{10}$ The systematic application of new techniques for virus identification in the present series most probably accounts for the increase in the overall diagnostic yield compared with our previous experience. ${ }^{9}$

Another important finding of the present study is that cultures of TBAS in intubated patients were helpful in determining the aetiology of pneumonia. Traditionally, TBAS cultures have not been considered specific enough to diagnose lower respiratory tract infections due to the presence of bacterial colonisation of both lower and upper airways. However, researchers from our institution ${ }^{29}$ and others ${ }^{30}$ have shown that, using quantitative cultures, the aetiology of bacterial pneumonia can be accurately ascertained in mechanically ventilated patients using TBAS cultures. In the present series we have confirmed the usefulness of TBAS cultures in immunocompromised patients, obtaining a diagnosis in $64 \%$ of intubated patients investigated. Further studies are needed to clarify the usefulness of TBAS and to compare it with other techniques such as blind $\mathrm{BAL}^{31}$ which also does not rely on a bronchoscopist and has a good diagnostic yield in immunocompromised patients. In contrast, PSB gave few positive results (24\%), many of them also achieved by BAL or FBAS. In only one case was PSB the technique that led to changes in treatment.

Bronchoalveolar lavage has been established as a reliable technique for the diagnosis of pulmonary infiltrates in the immunosuppressed host, specifically for detecting opportunistic infectious such as $P$ carinii and CMV and also for bacteria. ${ }^{92}$ In the present series most episodes of pneumonia caused by opportunistic pathogens were diagnosed by BAL. Although this technique has been criticised by some authors because the impact on survival is disappointingly poor, ${ }^{16} 1733$ BAL has a high diagnostic yield for infections and provides enough material to diagnose alternative noninfectious aetiologies. Specifically, in our population BAL provided the diagnosis in 10 cases of diffuse alveolar haemorrhage, one case of pulmonary toxicity due to bleomycin, and in one case of alveolar proteinosis.

Although TBB specimens are not routinely taken from immunocompromised patients in our centre, it has clear indications in specific cases such as those with nodular, localised or patchy infiltrates on the chest radiograph. In the present series TBB not only gave a specific diagnosis in six of the 11 patients in which it was performed, but also allowed a change in the treatment in four cases (two with pulmonary lymphoma, two with BOOP).

Compared with our previous series, ${ }^{9}$ the current diagnostic techniques employed increased the percentage of treatment changes from $31 \%$ to $46 \%$. However, there was no difference in mortality when patients with and without changes in treatment due to the results obtained with the different diagnostic procedures employed were compared. The lack of impact of invasive diagnostic techniques on survival has been claimed by some authors to be the major reason for not performing them in immunocompromised patients. ${ }^{16}$ Moreover, complications and morbidity secondary to bronchoscopy have been described in this setting. ${ }^{17}$ We suggest that the treatment of infectious pulmonary infiltrates in immunocompromised patients has a poor outcome because specific drugs are usually administered late in the evolution of the illness. In fact, we found a significant decrease in mortality in those patients with an infectious aetiology in whom an early diagnosis (7 days from the initiation of the pulmonary infiltrates) caused a change in treatment $(29 \%)$ compared with those in whom this diagnosis was obtained after 7 days ( $71 \%)$. Although it seems logical, based on these results, to recommend the early use of diagnostic techniques to improve the clinical outcome, further specifically designed studies are needed to try to answer this critical question. Confounding factors such as the judgement of clinicians in charge to try different treatments in the face of clinical deterioration might associate changes in treatment with worse prognosis in the present study.

Based on the results obtained, our recommendation is to use simple non-invasive 
procedures as a first step in the diagnosis of pulmonary infiltrates in the immunocompromised patient. These non-invasive procedures should include blood, sputum, and NPW cultures as well as TBAS in patients on mechanical ventilation. The use of noninvasive techniques provides the diagnosis in $44 \%$ of the cases and constitutes a good alternative in those patients with contraindications for a bronchoscopic exploration. Because of the importance of obtaining a diagnosis with minimum delay, BAL should always be performed when possible as this endoscopic procedure has a high diagnostic yield for both infectious and non-infectious aetiologies. The use of PSB could be avoided since it does not contribute to increasing the diagnostic yield of BAL. This rather expensive technique could probably be replaced by a more simple FBAS. Finally, provided no contraindications exist, selected patients should be submitted to transbronchial biopsies as a step before surgical procedures.

In summary, the use of both non-invasive and bronchoscopic procedures substantially increases the diagnostic yield of pulmonary infiltrates, causing changes in the empirical treatment in the majority of patients. Survival in patients who require a change in treatment seems to be higher when the specific diagnosis is established early in the course of the disease. However, further studies are needed definitively to confirm the importance of an early diagnosis on the clinical outcome in immunocompromised patients with pulmonary complications.

$\mathrm{AR}$ is supported by a clinical research grant from SEPAR and Hospital Clínic de Barcelona (grant 1999SGR00228 from the Comissionat per a Universitat i Recerca de la Generalitat de Catalunya). PJ is a research fellow from the Universidad Austral de Chile, Valdivia, Chile.

1 Ettinger NA, Trulock EP. Pulmonary considerations of organ transplantation. Am Rev Respir Dis 1991;144:433451.

2 Ewig S, Torres A, Riquelme R, et al. Pulmonary complications in patients with haematological malignancies treated at a respiratory ICU. Eur Respir f 1998;12:116-22.

3 Baughman RP. The lung in the immunocompromised patient. Infectious complications. Part 1. Respiration 1999; 66:95-109.

4 Tamm M. The lung in the immunocompromised patient. Infectious complications. Part 2. Respiration 1999;66:199207

5 Agustí C, Ramirez J, Picado C, et al. Diffuse alveolar hemorrhage in allogenic bone marrow transplantation. A postmortem study. Am $\mathcal{F}$ Respir Crit Care Med 1995;151: postmortem 1006 . 10 .

6 Agustí C. Diffuse alveolar hemorrhage in patients after bone marrow transplantation. Concerns in relation to diagnosis. 7 Bronchol 1998;5:95-7.

7 Robbins RA, Linder J, Stahl MG. Diffuse alveolar hemorrhage in autologous bone marrow transplant recipients. Am F Med 1989;87:511-8.

8 Crawford SW. Non-infectious lung disease in the immunocompromised host. Respiration 1999;66:385-95.
9 Xaubet A, Torres A, Marco F, et al. Pulmonary infiltrates in immunocompromised patients. Chest 1989;95:130-5.

10 Leland DS, Emanuel D. Laboratory diagnosis of viral infections of the lung. Semin Respir Infect 1995;10:189-98.

11 Whimbey E, Bodey GP. Viral pneumonia in the immunocompromised adult with neoplastic disease: the role of common community respiratory viruses. Semin Respir Infect 1992;7:122-31

12 Ridell SR. Pathogenesis of cytomegalovirus pneumonia in immunocompromised hosts. Semin Respir Infect 1995;10: 199-208.

13 Yousuf HM, Englund J, Couch R, et al. Influenza among hospitalized patients with leukemia. Clin Infect Dis 1997;24:1095-9.

14 Wendt CH, Weisdorf DJ, Jordan MC, et al. Parainfluenza virus respiratory infection after bone marrow transplantation. N Engl f Med 1992;326:921-6.

15 Mayaud C, Cadranel J. A persistent challenge: the diagnosis of respiratory disease in the non-AIDS immunocompromised host. Thorax 2000;55:511-7.

16 Dunagan DP, Baker AM, Hurd DD, et al. Bronchoscopic evaluation of pulmonary infiltrates following bone marrow transplantation. Chest 1997;111:135-41.

17 White P, Bonacum JT, Miller CB. Utility of fiberoptic bronchoscopy in bone marrow transplant patients. Bone Marrow Transplant 1997;20:681-7.

18 Murray PR, JA Washington II. Microscopic and bacteriologic analysis of expectorated sputum. Mayo Clin Proc 1975;50:339-44.

19 Wimberley NW, Bass JB, Boyd BW, et al. Use of a bronchoscopic protected catheter brush for the diagnosis of pulmonary infections. Chest 1982;81:556-62.

20 El-Ebiary M, Torres A, Fàbregas N, et al. Significance of the isolation of Candida species from respiratory samples in critically ill non-neutropenic patients. Am $\mathcal{F}$ Respir Crit Care Med 1997;156:583-90.

21 Hughes WT, Armstrong D, Bodey GP, et al. Guidelines for the use of antimicrobial agents in neutropenic patients with the use of antimicrobial agents in neutropenic patie

22 Niederman M.S. Is "crop" rotation of antibiotics the solution to a "resistant" problem in the ICU? Am f Respir Crit Care Med 1997;156:1029-31.

23 American Thoracic Society. Hospital acquired pneumonia in adults: diagnosis, assessment of severity, initial antimicrobial therapy, and preventive strategies. A consensus statement. Am $\mathcal{F}$ Respir Crit Care Med 1996;153:1711-25.

$24 \mathrm{McWhinney} \mathrm{PH,} \mathrm{Kibbler} \mathrm{CC,} \mathrm{Hamon} \mathrm{MD,} \mathrm{et} \mathrm{al.} \mathrm{Progress}$ in the diagnosis and management of aspergillosis in bone marrow transplantation: 13 year's experience. Clin Infect Dis 1993;17:397-404.

25 Denning DW. Therapeutic outcome in invasive aspergillosis. Clin Infect Dis 1996;23:608-15.

26 Von Eiff $M$, Roos N, Schulten R, et al. Pulmonary aspergillosis: early diagnosis improves survival. Respiration 995;62:341-7

27 Horwath JA, Dummer S. The use of respiratory-tract cultures in the diagnosis of invasive pulmonary aspergillosis. Am f Med 1996;100:171-8.

28 Maertens J, Verhaegen J, Demuynck H, et al. Autopsycontrolled prospective evaluation of serial screening for circulating galactomannan by a sandwich enzyme-linked immunosorbent assay for hematological patients at risk for invasive aspergillosis. F Clin Microbiol 1999;37:3223-8.

29 El-Ebiary M, Torres A, González J, et al. Quantitative cultures of endotracheal aspirates for the diagnosis of ventilator-associated pneumonia. Am Rev Respir Dis 1993; 148: $1552-7$.

30 Marquette $\mathrm{CH}$, Georges $\mathrm{H}$, Wallet $\mathrm{F}$, et al. Diagnostic efficiency of endotracheal aspirates with quantitative bacterial cultures in intubated patients with suspected pneumonia. Am Rev Respir Dis 1993;148:138-44.

31 Papazian L, Thomas P, Garbe L, et al. Bronchoscopic or blind sampling techniques for the diagnosis of ventilatorassociated pneumonia. Am $\mathcal{f}$ Respir Crit Care Med 1995;152:1982-91.

32 Stover DE, Zaman MB, Hajdu SI, et al. Bronchoalveolar lavage in the diagnosis of diffuse pulmonary infiltrates in the immunosuppressed host. Ann Intern Med 1984;101:17 .

33 Campbell JH, Blessing N, Burnett AK, et al. Investigation and management of pulmonary infiltrates following bone marrow transplantation: an eight year review. Thorax 1993; 48:1248-51 